\title{
UNA VISIÓN PSICONEUROINMUNOLÓGICA DE LA FIBROMIALGIA
}

\author{
TOMÁs ÁLVARO ${ }^{1}$ Y FRANCISCO TRAVER ${ }^{2}$ \\ ${ }^{1}$ Hospital Verge de la Cinta, Tortosa \\ ${ }^{2}$ Área de Salud mental del Hospital Provincial, Castellón de la Plana
}

\begin{abstract}
Resumen: El síndrome de fibromialgia (SFM) es el síndrome de dolor crónico que se encuentra con mayor frecuencia en medicina general. Junto a una miríada de alteraciones inespecíficas biológicas y sintomáticas, la evidencia sugiere un procesamiento anómalo del dolor a nivel central en ausencia de disfunción periférica y en ausencia de enfermedad. Alteraciones emocionales o psiquiátricas inciden sobre los sistemas nervioso, endocrino e inmune, que explican a través del eje psico-neuro-inmuno-endocrinológico un desequilibrio homeostático que se traduce en forma de disautonomía y disritmia y explica las alteraciones del sistema nervioso vegetativo, del ritmo circadiano, del sueño, hormonales e inmunes y abre un nuevo horizonte de manejo y tratamiento del SFM. Los tratamientos no medicamentosos capaces de equilibrar este triángulo homeostático van desde la terapia de comportamiento de enfoque cognitivo, la hipnoterapia, métodos de biofeedback electromiográfico, ejercicio físico moderado y acupuntura, sin olvidar una atención especial al restablecimiento del ritmo circadiano y a la mejora de la calidad del sueño.
\end{abstract}

Palabras clave: Síndrome de fibromialgia, psiconeuroinmunología, alteraciones del sueño, dolor, disautonomía.

\section{A psychoneuroimmunological view of fibromyalgia}

\begin{abstract}
Fibromyalgia syndrome (FSM) is the chronic pain syndrome most frequently found in general medicine. Along with a myriad unspecific biological and symptomatic alterations, the evidence points at anomalous pain processing at a central level, in the absence of peripheral dysfunction or illness. Emotional or psychiatric alterations affect the nervous, endocrine and immune systems which explains, through the psycho-neuro-immune-endocrinologic axis, the homeostatic imbalance that gives rise to dysautonomia and dysrhythmia and finally explains alterations of the vegetative nervous system, of the circadian rhythm and sleep, hormonal and immune rhythms, and opens a new horizon as to handling and treatment of FSM. Non-drug treatments, capable of balancing this homeostatic triangle, go from cognitive-behavior therapy to hypnotherapy, forms of electromyographic biofeedback, moderate physical exercise and acupuncture, not forgetting a special attention to re-establishing the circadian rhythm and to improve sleep quality.
\end{abstract}

Keywords: Fibromyalgia syndrome, psychoneuroimmunology, sleep disturbs, chronic pain, dysautonomia.

\section{EL SÍNDROME DE FIBROMIALGIA}

El síndrome de fibromialgia (SFM) se caracteriza por dolor generalizado que no se explica por otro diagnóstico (Abeles, Pillinger,

Recibido: 18-septiembre-2009; aceptado: 1-diciembre-2009

Correspondencia: Tomás Álvaro Naranjo, Jefe de Servicio de Patología, Hospital Verge de la Cinta, c/ Esplanetes 14, 43500 Tortosa (España).

Correo-e: talvaro.ebre.ics@gencat.cat
Solitar, y Abeles, 2007; Sharpe, 2006). Constituye un síndrome funcional, como el síndrome de fatiga crónica, el dolor torácico no cardíaco, el síndrome de hiperventilación, el síndrome de intestino irritable, y tantos otros que comparten como denominador común una definición sintomática de la entidad. La discusión de si se trata de un trastorno orgánico o psicógeno pivota entre la búsqueda de hallazgos patológicos, infructuosa hasta la fecha, y su consideración como no-enfermedad, carente de raíces bioló- 
gicas y fruto de una construcción social basada en la amplificación psicológica de sensaciones somáticas normales. Desde el punto de vista psiquiátrico suele ser considerada un trastorno de ansiedad, del estado de ánimo o somatomorfo.

Hasta la fecha la fisiopatología del SFM permanece poco clara y muchos clínicos se sienten incómodos con el tratamiento y el manejo de estos síndromes poco entendidos (Alegre de Miquel y Sellas Fernández, 2008; Rodero, García-Campayo, Casanueva y Buriel, 2009). A lo largo de la historia, el SFM se ha reconocido como una alteración denominada neurastenia que pone énfasis en la fatiga; dolor crónico, queja colectiva de la $2^{\mathrm{a}}$ mitad del $\mathrm{s}$. XX, $\mathrm{o}$ incluso histeria. Esta enfermedad ha sido bautizada como fibrositis, dolor crónico generalizado, encefalomiopatía miálgica y síndrome de dolor miofascial, hasta que el American College of Rheumatology adoptó el término de fibromialgia (Wolfe et al., 1990). Los criterios diagnósticos propuestos son dolor generalizado durante al menos tres meses e hipersensibilidad músculoesquelética, ligamentaria y tendinosa al dolor en once o más de 18 localizaciones específicas o puntos gatillo.

Según la Sociedad Española de Reumatología (2006), y una vez superada la idea del sobrediagnóstico, los datos epidemiológicos de la fibromialgia suponen una incidencia en mayores de 20 años del 2,4\% de la población, $0,2 \%$ hombres y $4,2 \%$ de las mujeres, lo que supone unos 700.000 pacientes en España, con un pico entre los 40-50 años. Es la tercera causa de consulta en reumatología y es una enfermedad con importantes repercusiones socioeconómicas.

Pero el SFM incluye una cantidad ingente de alteraciones asociadas al dolor y que con frecuencia se solapan con los de otras enfermedades reumáticas, entre las que Arias (2008) incluye fatiga, intolerancia al ejercicio, parestesias, sensación de hinchazón, fotofobia, fonofobia, migraña, cefalea tensional, trastornos visuales, sacudidas, aftas, sequedad de mucosas, disfunción de la articulación témporo-mandibular, mareos, vértigo, inestabilidad, trastorno de memoria, falta de concentración, ansiedad, crisis de angustia, depresión, colon irritable, cistitis, síndrome premenstrual, dismenorrea, síndrome de piernas inquietas, mioclono nocturno, fenómeno de Raynaud y taquicardias. Esto hace que el diagnóstico de SFM se realice cuando el paciente no puede ser encuadrado dentro de otra enfermedad, siendo especialmente frecuente encontrar sus síntomas en pacientes con lupus eritematoso sistémico (Buskila, Press, y Abu-Shakra, 2003; Wolfe et al., 2009) y artritis reumatoide (Wolfe y Michaud, 2009).

En relación con su fisiopatogenia, sabemos que las fibras musculares no tienen nociceptores, razón por la cual las enfermedades musculares degenerativas no resultan dolorosas. En el SFM, la biopsia muscular ha mostrado una amplia gama de hallazgos, inconstantes e inespecíficos, como fibras en sacabocados, atrofia de fibras tipo 2, hendiduras en la membrana basal, acúmulo de lipofucsina, mitocondrias con patrones irregulares o alteraciones de la microcirculación (Bengtsson, 2002). Sin embargo lo cierto es que hasta la fecha no se ha conseguido detectar biomarcador fiable alguno que permita un diagnóstico objetivo de esta enfermedad (Dadabhoy, Crofford, Spaeth, Russell y Clauw, 2008). Su etiología permanece desconocida hasta la fecha, si bien se han implicado un gran número de factores tanto ambientales como genéticos. En la actualidad se tiende a pensar que existen una serie de factores predisponentes, desencadenantes y perpetuantes de tipo biológico, psicológico y social (Sharpe, 2006). El espectro de factores incluye desde la personalidad, los malos tratos, la falta de apoyo social o el estrés vital, hasta infecciones víricas o lesiones físicas en el cuello, pasando por infecciones crónicas, anomalías inmunológicas, alteraciones del sueño o cambios neuroendocrinos. Todos estos hallazgos sugieren un importante papel de los factores psicológicos, conductuales y sociales.

Se discute también si el SFM es un síndrome primario o secundario. El diagnóstico se realiza cuando el paciente no presenta signos de otra enfermedad ya que, como se ha mencionado, un cuadro similar puede encontrarse asociado a lupus eritematoso sistémico, artritis reumatoide, pacientes con cáncer libre de enfermedad, esclerosis múltiple y otros. Igualmente es posible encontrar fibromialgia asociada a otros síndromes como migraña, síndrome 
del intestino irritable, dolor de la articulación temporo-mandibular, trastorno de ansiedad y depresión.

Las bases neurobiológicas del SFM constituyen un difícil terreno de estudio, en donde los estudios electromiográficos no ofrecen hallazgos, la biopsia de músculo es normal o muestra cambios inespecíficos, el EEG muestra aumento de la amplitud del ritmo alfa y descargas epileptiformes aisladas, la polisomnografía muestra una disminución y alteración de estadios II, III, IV con intrusión de ritmos alfa (sueño no reparador), el líquido cefalorraquídeo puede mostrar aumento de aspartato, glutamato y substancia $\mathrm{P}$, en plasma se observa descenso de serotonina y Nad, con aumento de IL 1 y 4, estudios endocrinológicos han objetivado disminución de cortisol en orina, aumento de la respuesta a ACTH y déficit de $\mathrm{GH}$, estudios de neuroimagen funcional han comunicado disminución de flujo en corteza dorsolateral prefrontal, corteza parietal superior, cabeza del caudado, tálamo y tegmento pontino inferior, la evaluación psiquiátrica frecuentemente encuentra depresión, fobias simples, distimia, crisis de angustia, antecedentes de abusos sexuales y malos tratos infantiles y los estudios genéticos indican un riesgo elevado para familiares de primer grado.

El pronóstico del SFM es variable, con un curso crónico y fluctuante y un $50 \%$ de remisión parcial o completa a los 2-3 años (Sharpe, 2006). Parecen indicar un pronóstico adverso la enfermedad de larga duración, la presencia de síntomas más intensos, mayor edad, depresión, falta de apoyo social y fuerte creencia en causas físicas. Con respecto al tratamiento, se han usado con distinta suerte la amitriptilina (un antidepresivo de carácter serotoninérgico) que mejoraba el estado de ánimo y el sueño de estas pacientes aun a dosis subterapéuticas, también el tetrazepan - un relajante muscular (en realidad una benzodiacepina) - que si bien mejoraba el sueño parece que empeoraba la fatigabilidad y las contracturas. Más adelante se han ensayado los inhibidores selectivos de la recaptación de la serotonina (ISRS) como la fluoxetina, citalopram, sertralina y paroxetina con distinta suerte según los autores. Recientemente se ha recomendado el uso de los antidepresivos duales (venlafaxina, duloxetina) que parece que unen a su perfil antidepresivo una cierta acción antiálgica. El último fármaco puesto a punto es la pregabalina, un neuroprotector - antiglutaminérgico - que mejora el sueño de estos pacientes así como los síntomas ansiosos. Vale la pena señalar en este momento que los únicos tratamientos que han mostrado cierta eficacia son los psicofármacos en combinación con determinados consejos hipocráticos como la recomendación de la natación, el fortalecimiento del tono muscular a base de caminatas y la acupuntura (Targino et al., 2008) que parecen señalar ciertos beneficios en pacientes individuales a pesar de que no se dispone de estudios controlados frente a esta tecnología mal conocida aun en nuestra medicina.

Después de múltiples intentos de comprender la enfermedad, la comunidad médica sigue debatiendo si el SFM tiene un origen orgánico o psicosomático, y hasta se han propuesto aproximaciones filosóficas que permitan comprender su origen, el efecto de la relación terapeuta-paciente y el papel de los factores sociales en ella (Hazemeijer y Rasker, 2003). Junto con determinadas propuestas que cuestionan la propia definición de enfermedad en sí, como constructo social o bien como conceptualización de la propia medicina, determinados autores (Ehrlich, 2003) plantean que el dolor que presentan los pacientes es real, lo que les aleja de las conceptualizaciones clásicas sobre la histeria pero cuestionan el constructo nosográfico en sí, es decir a la propia fibromialgia. Lo mismo supone Bornhauser (2005) que incluso llega a listar una serie de criterios que hacen «sospechosa» de no serlo cualquier enfermedad que los cumpla (véase la Tabla 1).

A estos criterios nosotros añadiríamos una décima condición, y es que con frecuencia los pacientes se organizan en asociaciones vindicativas y hostiles frente a cualquier hallazgo que ponga en cuestión la ortodoxia del grupo que suele estar relacionada con el miedo al estigma psiquiátrico. Lo cierto es que se trata de una enfermedad con una miríada de alteraciones bioquímicas, fisiológicas, sintomáticas y etiológicas, devastadora para quien la sufre y de la que verdaderamente hasta la fecha no se dispone de un marco conceptual que permita enten- 
Tabla 1. Criterios sobre el síndrome de fibromialgia (según Bomhauser, 2005)

1. Los cuadros clínicos en cuestión no han sido objetivamente demostrados.

2. No se cuenta con tests diagnósticos o marcadores biológicos específicos.

3. No existe un consenso científico acerca de etiología, patogénesis y patofisiología.

4. Las respectivas concepciones de medicina tradicional y medicina alternativa son discrepantes y en ocasiones, hasta contradictorias.

5. No existe una terapia específica, probada en estudios controlados aleatorizados.

6. El diagnóstico goza de gran popularidad entre los pacientes.

7. Hay una animada discusión en la prensa de difusión y los medios de masas.

8. Se observa una alta recurrencia a curanderos y métodos marginales.

9. La medicina académica sigue escéptica frente a terapias sin prueba de eficiencia en estudios controlados.

derla y mucho menos de un tratamiento y un manejo eficaz.

\section{EL SÍNDROME DE FRIBROMIALGIA COMO TRASTORNO PSICÓGENO}

La comorbilidad del SFM con determinados trastornos psiquiátricos ha sido señalada con tozuda insistencia apuntando hacia un hecho, que más que resolver añade un dilema más, puesto que hace difícil la discriminación entre aquellos que sostienen que los trastornos psiquiátricos o psicológicos del SFM son reactivos al dolor crónico o los que piensan que les preceden y aun que forman parte de la plasticidad de la enfermedad. Para nosotros sin embargo la coexistencia de síntomas psíquicos y orgánicos no es sino la demostración de que ambos registros se encuentran enlazados en lo que en este artículo denominamos una hipótesis psiconeuroinmunoendocrinológica de la fibromialgia: lo relevante no es si los síntomas mentales son secundarios o primarios a la enfermedad en sí, sino el efecto de «arrastre» mutuo que psique y soma en su continua homeostasis circular propician en el organismo vivo. Las evidencias de la comorbilidad están bien establecidas, si bien, como suele suceder en psiquiatría, pareciera como si las entidades psiquiátricas abandonaran su carácter de entidades discretas y se diluyeran a favor de una «macedonia» de síntomas. Estos parecen cada vez más proceder de un fondo timopático común del que ya se tienen también algunas evidencias genéticas (Giesecke et al., 2005) (Cohen, Buskila, Neumann, y Ebstein, 2002) (Offenbaecher et al., 1999) aunque em- píricamente ya habían sido descritas por López Ibor $(1966,1972)$ como «equivalentes depresivos» o por el constructo más reciente de «ansiedad subumbral». Ansiedad y depresión tendrían pues un origen común: un magma esencial de donde procederían diversos síntomas, así la ansiedad se asociaría de forma más usual con el dolor y la depresión con la fatiga.

Los estudios anteriormente citados (Giesecke et al., 2005; Cohen et al., 2002 ; Offenbaecher et al., 1999) vienen a replicar en este caso que existe una relación entre la existencia de ciertos polimorfismos en el gen transportador de la serotonina (HTPLR) y las entidades psiquiátricas, desde el alcoholismo hasta la esquizofrenia pasando por la ansiedad y la depresión. Al parecer existen dos polimorfismos de este gen, uno corto (s) y otro largo (1). Los sujetos homozigóticos que tienen dos copias de este polimorfismo corto (s) tienen más vulnerabilidad psiquiátrica que los heterozigóticos para este rasgo o los que tienen dos copias del polimorfismo largo (1), algo que se conoce desde hace mucho tiempo y que Lesch et al. (1997) investigaron desde un punto de vista evolutivo pero que aún requerirá nuevas conceptualizaciones que expliquen este fenómeno y sus relaciones con la psicopatología humana.

No es raro pues que el SFM haya sido relacionado con múltiples estados psiquiátricos, yendo a la cabeza la depresión, una auténtica respuesta inmune compleja donde las citocinas actúan como mecanismo causal (Reiche, Morimoto, y Nunes, 2005). Alfici Sigal Landau (1989) consideran que la fibromialgia es una variante de un desorden depresivo primario, mientras que otros piensan que la variable cri- 
tica es la distorsión cognitiva - el pensamiento catastrófico y pesimista - ligado a la depresión (Hassett, Cone, Patella, y Sigal, 2000). Hudson y Pope (1989) en un planteamiento dimensional proponen que el SFM sea contemplado como un estado más de un amplio abanico del espectro afectivo. No podían faltar tampoco los que creen que es un síntoma más del trastorno de estrés postraumático (TEP) basándose en los antecedentes relatados por los pacientes entre los que se encuentran tasas diversas de abuso sexual o explotación infantil dependiendo de si son americanos o europeos, sesgos que se encuentran casi siempre en la literatura psiquiátrica cuando comparamos las muestras de pacientes americanos y los de este lado del mar (Roy-Byrne, Smith, Goldberg, Afari y Buchwald, 2004). Así y todo en una muestra europea se encuentra 5 veces más frecuencia de antecedentes de abusos infantiles en comparación con la población general (Fietta, 2004).

En lo que todos los investigadores están de acuerdo es que no estamos delante de una patología uniforme o homogénea, por eso no podemos hablar de una «personalidad fibromiálgica» si bien se han descrito rasgos diversos asociados con el SFM, los más nombrados de los cuales son la alexitimia y la rabia (Sayar, Gulec, Topbas y Kalyoncu, 2004). La alexitimia es un rasgo de personalidad (Sifneos, 1973) que se ha encontrado fuertemente asociado a la patología psicosomática (Rueda y Pérez-García, 2007), se trataría de personas que encuentran dificultades en nombrar o verbalizar sus emociones. Junto a la alexitimia se ha encontrado fuerte comorbilidad sobre todo con tres trastornos de la personalidad: el limite, el obsesivocompulsivo y el depresivo, aunque lo cierto es que hay pocos informes acerca de la coexistencia de trastornos de la personalidad con la fibromialgia, siendo el trastorno límite el más referido. Por el contrario el trastorno evitativo de la personalidad está menos representado entre la población con SFM que en la población general (Fietta, 2004).

Lo seguro es que el constructo psiquiátrico «trastorno somatomorfo» es el más plausible para clasificar esta enfermedad pero se nos queda corto revelando así la insuficiencia tanto del constructo nosológico (Carmona et al.,
2007) como de una teoría inexistente aunque nos impide explicar como lo mental se transforma en orgánico y a la inversa. El término somatomorfo o «somatización» es sólo descriptivo y por tanto ateórico y ha seguido el mismo destino que la idea de la patología psicosomática: murió apenas ser enunciada a falta de una teoría que empareje los estados mentales con los sufrimientos somáticos. Goicoechea (2008) plantea que la jaqueca (el dolor somatoforme o neuropático) pueda ser una respuesta exagerada del sistema nociceptivo ante una señal neutral que desencadena la cascada del dolor. En este sentido el dolor sería la puesta en marcha de un programa filogenético destinado a la autolimitación del daño y que no se debería a una inflamación o daño muscular real sino a la activación de los nociceptores «durmientes o silenciosos». El mismo Goicoechea ha señalado una estructura psíquica fóbica en la mayor parte de sujetos que presentan jaquecas.

Desde el punto de vista de la personalidad, los autores tampoco acaban de ponerse de acuerdo pues se trata de personas difíciles de clasificar con nuestros instrumentos actuales, personas temerarias y temerosas simultáneamente, no son personas que presenten fobias clínicas pero mantienen una multitud de temores racionalizados tras una máscara de evitación e hipercontrol (Felipe y Ávila, 2008). El carácter fóbico es pues una atmósfera de negación o racionalización de temores o repugnancias que es muy similar al constructo «ansiedad subumbral» que remite del mismo modo a una ansiedad no clínica que permanece así ajena a la consciencia pero que se manifiesta sobre todo a través de una fácil activación adrenérgica: se trata de personas que aparecen siempre como apuradas o hiperactivas sin que presenten signos de ansiedad clínica. Del mismo modo tampoco presentan - los más adaptados de entre ellosevitación fóbica, como si representaran nosológicamente una transición entre lo obsesivo y la fobia en sí, una especie de terreno de nadie donde quedan instalados de por vida sin ser casi nunca detectados debido a lo intangible del término aunque este equilibrio está destinado al deterioro progresivo. Este tipo de personalidades son adherentes y pegajosas, perseveran- 
tes, concienzudas, tozudas, a veces hipercríticas, querulantes u hostiles pero siempre rígidas o hipercontroladoras y con una escotomización de su campo de intereses que resulta del ejercicio constante de la evitación como modelo de afrontamiento. El rasgo border-line «sentimiento crónico de vacío» parece que se adapta a un sentimiento permanente de evaluación del sí mismo característico de algunos pacientes que construyen relaciones de dependencia o codependencia con los suyos como mecanismo de oponerse a cualquier cambio que implique entradas o salidas en el campo social u otras novedades.

El constructo de Cloninger «evitación del daño» (Cloninger, 1987) es un endofenotipo, es decir algo que se encontraría a medio camino del genotipo y del fenotipo y es probablemente el rasgo temperamental que mejor se acoplaría para medir a través del inventario del temperamento y el carácter TCI-R. Las personas que puntúan alto en este rasgo se caracterizarían por preocupación y pesimismo, temor a la incertidumbre, timidez social y fatigabilidad fácil. Como puede observarse esta tipología no equivale linealmente al «trastorno de personalidad por evitación» pero se solapa con él. El constructo clásico que propone Goicoechea en este sentido está cargado de pruebas empíricas que lo avalan y que participaría de un suelo común con todo el cluster $\mathrm{C}$, la hipersensibilidad social y la evaluación temerosa del mundo junto con la desconfianza - que en algunos casos podría rayar lo paranoide y que podrían ser rasgos comunes en los pacientes afectos de dolor neuropático. El carácter fóbico es un trastorno más adaptado que el trastorno de evitación y seguramente interfiere menos en la vida diaria, personal o laboral de los sujetos que lo sufren.

En definitiva y utilizando palabras de Albert Einstein, el mundo de hoy enfrenta problemas que no pueden ser resueltos pensando en la misma forma en que se pensaba cuando fueron creados. Con ese espíritu nosotros proponemos aquí un nuevo abordaje conceptual y propuesta terapéutica del SFM, que permitan avanzar en el proceso de conocimiento y tratamiento de esta enfermedad. Y para conseguirlo la herramienta propuesta es la psiconeuroinmunología (PNI).

\section{AUTOREGULACIÓN PSICOFISIOLÓGICA DEL ORGANISMO}

Los sistemas nervioso (SN), endocrino (SE) e inmune (SI) constituyen los sistemas de control homeostático que sincronizados mantienen el equilibrio fisiológico interno del organismo, a través de una nutrida red de relaciones donde $\mathrm{CRH}$ tiene efectos directos sobre las células del SI, el hipotálamo regula el SNA y el SE e incluso el cerebro participa sintetizando citocinas a través de diferentes células, como la glía, astrocitos y neuronas. Por otro lado, también el SI modula el SN (Glaser y Kiecolt-Glaser, 2005) ya que neuronas y células gliales tienen receptores para citocinas (IL-1, IL-2, TNF-a, IFN-g) y los macrófagos producen factores neurotróficos, mientras que las células inmunes son a su vez productoras de hormonas y neurotransmisores (Blalock, 2005).

El SN recibe el impacto de estímulos ambientales, tales como factores psicosociales y diferentes estresores, que actúan sobre el cerebro poniendo en marcha una serie de señales químicas que alcanzan el SI, ya sea de forma directa o por mediación del SE. Estímulos cognitivos endógenos durante la reconstrucción de la memoria pueden activar circuitos neurales que movilizan la respuesta de estrés en ausencia del trauma original (Misslin, 2003). En sentido inverso el SI detecta estresores infecciosos o inflamatorios a los que responde con la secreción de diversas citocinas que alcanzan el cerebro y modulan el comportamiento. Dicho diálogo está modulado por los neurotransmisores del $\mathrm{SN}$, las hormonas del SE y las citocinas del SI, auténticas moléculas de la información que en última instancia enlazan psique y soma, por lo que es posible vislumbrar ya como diferentes estresores son capaces de producir un efecto sobre diversos órganos y sistemas con capacidad de producir enfermedad.

El sueño y el estado inmune se relacionan profundamente ya que las citocinas segregadas cambian la cantidad y el perfil del sueño REM/ noREM y problemas de sueño se asocian a infección, fibromialgia, cáncer y depresión. En particular parece ser la célula glial, la célula inmune del cerebro, la que responde a la substancia $\mathrm{P}$ tras un estímulo traumático o inflama- 
torio (Marriott, 2004), substancia P que además de estar alterada en el SFM ha sido implicada en la patofisiología de desórdenes inflamatorios así como alteraciones emocionales, constituyendo un auténtico eslabón de enlace mentecuerpo (Rosenkranz, 2007). Diversas citocinas (TNF, IL-1, IL-6) afectan el cerebro y el comportamiento a través de la hipertermia, nocicepción, anorexia, fatiga, adormilamiento y alteraciones del sueño, disminución del comportamiento social y locomotor, depresión y otras. De esta manera un proceso inflamatorio afecta el metabolismo energético y finalmente el comportamiento y la conducta.

Estímulos estresantes de diversa índole ponen en marcha una serie de mecanismos dependientes fundamentalmente de glucocorticoides y catecolaminas capaces de producir lesión inflamatoria sobre sistema nervioso periférico (SNP) y SNC (Garcia-Bueno y Leza, 2008). Los estados afectivos negativos debilitan la respuesta inmune (Rosenkranz et al., 2003) y colocan al individuo en una situación de riesgo de padecer enfermedades infecciosas, cardiovasculares, autoinmunes y tumorales (Barak, 2006). Así pues, el SN y el SI se comunican a través de un lenguaje bioquímico común, estableciendo un circuito bidireccional soportado por moléculas de información, citocinas, neurotransmisores, hormonas y sus correspondientes receptores. Este triángulo de equilibrio homeostático explica a nivel fisiológico como la mente puede influir el cuerpo.

El campo de la psiconeuroinmunología tiene como objeto de estudio y experimentación la autorregulación psicofisiológica del organismo y los mecanismos de interacción entre mente y sistemas responsables de mantener la homeostasis (Ader y Cohen, 1975). Esta comunicación utiliza un lenguaje bioquímico a través de mediadores como hormonas, neurotransmisores y citocinas y explica como el psiquismo modula la función inmune. Factores psicosociales, el estrés emocional y físico y también el comportamiento inciden sobre el $\mathrm{SN}$, que a través de su comunicación privilegiada con el SE y SI mantienen en equilibrio homeostático el organismo y determinan su estado de salud o enfermedad. Mientras que el estrés físico afecta el hipotálamo y activa la vía autonómica para conseguir la liberación de catecolaminas por parte de la médula adrenal que acaba actuando sobre el SI, el estrés emocional afecta el eje hipotálamo-hipófiso-corticosuprarrenal (HHC) para acabar estimulando la corteza suprarrenal y la secreción de glucocorticoides que tendrán su efecto sobre el SI. Cada tipo de estrés produce un patrón específico de impacto sobre el SI. La suma de varios factores, junto a la edad, dieta y otros, produce el patrón inmune propio de cada individuo, del que depende su estado de salud o de enfermedad, y en el último caso qué tipo de enfermedad está propenso a sufrir. Tanto el envejecimiento como el estrés afectan el SI produciendo una disregulación funcional con repercusiones sobre la salud. Experiencias estresantes en épocas muy precoces de la vida e incluso anteparto alteran las respuestas del SN y del SI con efectos medibles en la respuesta vacunal en la adolescencia, en los niveles de cortisol en el adulto y en las alteraciones cardiovasculares y metabólicas del anciano (Graham, Christian, y Kiecolt-Glaser, 2006), concediendo tanto a dichas experiencias precoces como al estrés un importante papel en la consideración de procesos del tipo del SFM.

\section{UNA VISIÓN \\ PSICONEUROINMUNOLÓGICA DE LA FIBROMIALGIA}

Se ha comparado el SFM con el estado mantenido del atleta preparado para salir en carrera, con todo su potencial cardiaco, vascular y muscular a punto y preparado para el máximo esfuerzo, pero en el que el juez no da el pistoletazo de salida. En la persona aquejada de SFM dicho estado simpático mantenido acaba produciendo un estado de desequilibrio homeostático y dolor. Este enfoque podría armonizar las diferentes propuestas para entender esta enfermedad, que incluyen la del síndrome metabólico, el modelo de sensibilización nerviosa central, el modelo de hipersimpaticotonía y otras. El dolor crónico resultante lleva consigo síntomas de fatiga, alteraciones del sueño, molestias físicas, alteración de la función mental y depresión (Chapman, Tuckett, y Song, 2008), todas ellas presentes en el SFM. 
Proponemos la consideración del SFM como un desequilibrio homeostático. Cualquier tipo de agresión al organismo en forma de estrés físico o psicológico supone una respuesta biológica defensiva que implica a los SN, SE y SI, que constituyen el sistema de equilibrio homeostático del organismo. Tras un período de respuesta, lo normal es que se alcance de nuevo un estado de equilibrio, sin embargo, de forma anómala, también es posible que se produzca un estado de disregulación, de especial importancia en situaciones de dolor crónico (Chapman et al., 2008). Entonces se produce una disfunción prolongada en la capacidad del sistema de recuperar tanto sus niveles normales como su relación normal con los otros sistemas, y por supuesto, un estado anómalo de respuesta a otros posibles estímulos. El SFM supone la alteración del sistema de respuesta al estrés, por lo que afecta a cada uno de sus componentes: a) al sistema nervioso autónomo (SNA) a través fundamentalmente de la variabilidad del latido cardíaco, produciendo una disautonomía; b) alteración del sueño y endocrina del eje HHC; y c) disritmia, con afectación del SI. Los cambios asociados a estos tres sistemas explican y dan coherencia a la gran cantidad de anomalías bioquímicas y fisiológicas de estos pacientes.

\section{ALTERACIÓN DEL SISTEMA NERVIOSO AUTÓNOMO: DISAUTONOMÍA}

El término disautonomía engloba una amplia variedad de trastornos como intolerancia ortostática, cefalea vascular, prolapso de la válvula mitral o colon irritable, que presentan como común denominador una alteración funcional del SNA. Entre estos cuadros se incluyen a su vez el síndrome de fatiga crónica y muy particularmente el SFM (Solano et al., 2009).

Puesto que el SNA representa la interfaz automática entre funciones mentales y corporales, constituye el eslabón que transmite de forma inmediata el papel de las emociones. Estas alcanzan la corteza cerebral y el área límbica, que irradia el estímulo hacia las zonas de tálamo, hipotálamo, tallo cerebral y médula espinal que controlan el SNA (Martinez-Lavin, 2007). En particular el SN simpático ha sido implicado en la patogenia de síndromes de dolor crónico a través de un estado hiperadrenérgico mantenido que propicia gracias a la neuroplasticidad el establecimiento de conexiones anómalas entre el SNS y las fibras nociceptivas, originando así un síndrome de dolor neuropático. La hiperactividad simpática de las pacientes con SFM ha invitado al intento de controlar el dolor mediante bloqueo simpático, ya sea de tipo quirúrgico (Bengtsson y Bengtsson, 1988) o médico (Wood, Kablinger, y Caldito, 2005).

Una aproximación de mayor impacto viene dada por el estudio de la variabilidad de la frecuencia cardiaca (HRV) como índice de la influencia del SN parasimpático sobre el nodo sinusal. La variabilidad cardiaca es un reflejo directo del tono vagal, por lo que transmite directamente un reflejo del estado del SNA así como de las funciones del comportamiento, emocionales y cognitivas que se encuentran asociadas con él (Beauchaine, Gatzke-Kopp, y Mead, 2007). El estrés intenso o el trauma, especialmente en estadios tempranos de la vida, produce una disminución permanente de la variabilidad cardiaca (Porges, 1992), que conlleva una menor capacidad de adaptación ante futuros estresores. El efecto de la hiperactividad simpática de los pacientes con SFM sobre HRV ha sido ampliamente investigado (Cohen et al., 2000; Martinez-Lavin, Hermosillo, Rosas, y Soto, 1998; Ulas et al., 2006; Furlan et al., 2005), y ha mostrado una paradójica situación de hiperactividad simpática persistente con hiporeactividad al estrés, que se explica debido a que la hiperestimulación crónica de los receptores beta-adrenérgicos suponen una desensibilización y regulación a la baja de dichos receptores. Y todavía de mayor interés resulta el hecho de que la hiperactividad simpática nocturna y la subsecuente alteración de la HRV se asocian a alteraciones del sueño (Otzenberger et al., 1998), alteraciones que resultan características de los y las pacientes con SFM, con un incremento de los episodios de despertar nocturno y un sueño poco reparador (Kooh et al., 2003), que vienen a sumarse a las alteraciones del sueño dependientes de los cambios del SE y SI (ver más adelante) de estos pacientes. 
Las alteraciones del sueño están siendo implicadas de forma creciente en el SFM (Martínez y Cassol, 2008), con somnolencia y cansancio, sueño fragmentado y no reparador, con frecuentes despertares nocturnos y a menudo disturbios de la respiración, como apnea del sueño y síndrome de resistencia de las vías aéreas superiores (Germanowicz, Lumertz, Martinez, y Margarites, 2006), constituyendo la deprivación selectiva de sueño profundo una causa conocida de dolor músculo-esquelético. Estas alteraciones han sido relacionadas con una disminución de los niveles de serotonina y triptófano, con un aumento de TNF alfa y de IL6 y con una reducción de hormona del crecimiento (Germanowicz et al., 2006). Dentro de las alteraciones del SN, se han comunicado numerosas alteraciones funcionales en algunas áreas cerebrales en pacientes con SFM, incluyendo hiperperfusión vascular en las áreas encargadas de diferenciar la intensidad del dolor, región parietal bilateral, incluyendo cortex postcentral y hipoperfusión en las áreas encargadas de discriminar las respuestas emocionales al dolor, región temporal anterior izquierda (Guedj et al., 2008) y disfunción del hipocampo (Emad et al., 2008). Gracias al fenómeno de la neuroplasticidad, la sobreestimulación crónica de fibras aferentes nociceptivas acaba produciendo hiperalgesia a través de dos vías, una la expansión de los campos receptivos y la consecuente reorganización de tallo cerebral y región cortical y otra a través del estímulo de neuropéptidos excitadores (Barr, Barbe, y Clark, 2004). Múltiples estudios recientes confirman la presencia de cambios microestructurales en el cerebro de pacientes con SFM (Emad et al., 2008; Lutz et al., 2008)

Por otro lado, tanto en animales como en humanos, los estudios muestran que el dolor altera la fisiología del sueño pero sobre todo que las alteraciones del sueño producen dolor músculo-esquelético (Moldofsky, 2008). Ello abre la puerta a enfocar la mejoría de la calidad del sueño en el SFM como una meta terapéutica principal, a favor de la cual se van sumando evidencias (Caruso, Sarzi Puttini, Cazzola, y Azzolini, 1990; Scharf, Baumann, y Berkowitz, 2003).

\section{ALTERACIÓN DEL EJE HIPOTÁLAMO- HIPÓFISO-CORTICOSUPRARRENAL Y DEL SISTEMA ENDOCRINO}

Los sistemas nervioso y endocrino colaboran estrechamente en la respuesta de estrés, implicando en su lenguaje bioquímico múltiples substancias como la hormona liberadora de corticotrofina $(\mathrm{CRH})$, la adrenalina, la noradrenalina, las beta endorfinas, etc., que actúan como neurotransmisores a nivel del $\mathrm{SN}$ y como hormonas sobre el SE. Los glucocorticoides, junto con las catecolaminas, constituyen las más importantes influencias reguladoras del SI. La médula adrenal, un órgano endocrino, constituye la expresión funcional del SNS. Las catecolaminas segregadas aumentan la frecuencia cardiaca, la respiración, contraen los músculos y afectan la musculatura vascular de diferentes órganos, incluyendo la musculatura. Las implicaciones del eje HHC han sido extensamente estudiadas en pacientes con SFM (Adler y Geenen, 2005), demostrando un grado variable de reducción en la actividad neuroendocrina, que naturalmente actúa de forma coordinada con el SNA. La reducción de la actividad del eje HHC, tal y como ocurre tras la retirada de tratamiento corticoideo o en enfermedad de Addison, a menudo se acompaña de síntomas de fatiga, depresión, mialgia y alteración del sueño, mientras que lo contrario también se ha observado, es decir, reducción de la fatiga e incremento de la energía y el bienestar con el incremento en los niveles de glucocorticoides (Jacobs et al., 2001). Por otro lado la actividad del eje HHC también está relacionada con la percepción del dolor, estimulando su activación la liberación de beta-endorfina, además de corticotropina y cortisol.

Los pacientes con SFM muestran síntomas similares a disfunción endocrina, como hipotiroidismo o insuficiencia adrenal o de hormona de crecimiento $(\mathrm{GH})$. En estas personas los niveles de actividad basal del eje HHC tienden a mantenerse dentro de límites normales. Sin embargo bajo condiciones de estrés tienden a mostrar una reducción en la respuesta de la función endocrina, especialmente de cortisol y hormona de crecimiento (Adler y Geenen, 2005), cambios que parecen estar en relación 
con la función hipotalámica, y no con la glándula adrenal o la hipófisis. La marcada hipersecreción de la hormona corticotropa (ACTH) en respuesta a estresores agudos ha sido interpretada como indicativa de hiposecreción crónica de CRH (Geenen, Jacobs, y Bijlsma, 2002). Y un número creciente de evidencias apoyan la hipótesis de que el sistema homeostático del organismo puede ser alterado a expensas de una respuesta inadecuada por parte del SE ligada a diferentes situaciones de estrés, como ocurre en la regulación epigenética del receptor de glucocorticoides tras abuso infantil (McGowan et al., 2009), tan frecuente en el SFM.

\section{ALTERACIÓN DEL SISTEMA INMUNE: DISRITMIA}

De la misma manera que el $\mathrm{SN}$ representa el primer agente de detección y defensa contra amenazas procedentes del medio ambiente externo, el SI realiza el mismo papel para el medio ambiente interno. La liberación de citocinas proinflamatorias a partir de un foco infeccioso, inflamatorio, tumoral, un traumatismo o un estímulo emocional o psicosocial, produce un efecto sobre el cerebro a diferentes niveles (Dunn, 2006), que en principio persigue el control del estímulo nocivo. Una vez conseguido el SI debe detener su respuesta para volver a la normalidad fisiológica homeostática o alostática. Si el SI no detiene su respuesta, ya sea porque el estímulo continúe, porque el SNC no detenga el proceso o porque el SI no ponga en marcha sus mecanismos de feedback negativo, tanto internos como de respuesta al SN, entonces se producirá una cronificación que alimenta el bucle de más citocinas, más estímulo cerebral y por tanto más estímulo del SI.

El SI regula las funciones del SNC relacionadas con el dolor (Watkins y Maier, 2005). Tanto las células inmunes activadas como las células gliales, en realidad la representación modificada del SI en el cerebro, desempeñan un papel fundamental en la modulación de la función neuronal (Watkins, Hutchinson, Milligan, y Maier, 2007). Son las células de tipo glial de la médula espinal las que se encargan de amplificar el dolor, modulando la excitabilidad de las neuronas espinales en respuesta a la inflamación o la infección. En condiciones patológicas, la activación glial produce un estado de hiperalgesia mediado por la consecuente liberación de citocinas proinflamatorias (Wieseler-Frank, Maier, y Watkins, 2005a, 2005b). La liberación de citocinas proinflamatorias por parte de microglia y astrocitos no solo actúan como mediadores de dolor en el área del trauma o la infección, sino que pueden extender la sensación de dolor a territorios vecinos sanos e incluso al hemicuerpo contrario al lugar del estímulo (Wieseler-Frank, Maier, y Watkins, 2004).

La activación del SI influye poderosamente sobre el proceso del sueño, tanto espontáneo como bajo condiciones inflamatorias, a través fundamentalmente de la IL1 y el TNF alfa (Krueger, 2008) a la vez que produce un síndrome clínico similar al SFM. Sin embargo las citocinas proinflamatorias producen un incremento del sueño; ciertamente los pacientes con fibromialgia sufren alteraciones de los niveles de citocinas proinflamatorias, especialmente IL8 y TNF alfa (Wang, Moser, Schiltenwolf, y Buchner, 2008), pero especialmente lo que muestran es una anómala secreción en forma de picos de las citocinas. Mientras que la secreción de hormonas suele obedecer a ritmos periódicos, la de citocinas lo hace en forma de estallidos ya sean únicos o dobles, con una duración entre picos de 110 minutos. En pacientes con SFM lo que se observa es un desequilibrio entre la secreción de citocinas proinflamatorias (que predominan en el período de sueño en personas sanas) y antiinflamatorias (que predominan en los pacientes con SFM), especialmente IL10, que explica las continuas interrupciones del sueño de estos pacientes (Togo et al., 2009). Curiosamente, esta alteración en el ritmo de secreción de las citocinas ocurre en el insomnio crónico, lo que ha llevado a considerar las alteraciones del sueño del SFM como una forma particular de insomnio. En él se comparten a la vez una hipersecreción durante el día de IL6 y TNF alfa, responsables de la fatiga, junto a un incremento de IL10 durante la noche, que propicia un sueño poco reparador, ambas propias de un patrón circadiano alterado (Vgontzas et al., 2002). Mientras que el factor liberador de la hormona de crecimiento (GH-RH) está aso- 
ciado con el sueño profundo no REM, la propia GH lo está con la fase REM, equilibrando entre ambos el anabolismo tisular durante el sueño (Obal y Krueger, 2004). La secreción de GHRH depende de la región preóptica del hipotálamo anterior, precisamente la misma zona que se ocupa de la regulación del sueño (Peterfi, Makara, Obal, y Krueger, 2009). Finalmente, el SI de hombres y mujeres funciona de manera ligeramente diferente, resultando cambios tanto del sueño como inmunes durante las diferentes fases del ciclo menstrual, lo que parece tener especial significado en el SFM. Especialmente la IL1 aumenta durante la fase luteínica, es producida por la glía y desarrolla un importante papel en la promoción del sueño (Moldofsky, 1995).

\section{CAMBIO DE PARADIGMA EN EL SÍNDROME DE FATIGA CRÓNICA}

E1 SFM no es una alteración músculo-esquelética ni una enfermedad inflamatoria sistémica, como tampoco es una alteración mental ni una anomalía genética. Proponemos una visión psiconeuroinmunológica del SFM capaz de englobar y de dar coherencia a la ingente cantidad de alteraciones biológicas y sintomáticas de estos pacientes. Dichas alteraciones incluyen: a) disautonomía, dependiente de la alteración del SNA, con alteración de la variabilidad de la frecuencia cardiaca y todas sus consecuencias; hiperactividad simpática e hiporeactividad al estrés; alteraciones del sueño con incremento de los episodios de despertar nocturno y sobreestimulación crónica de las fibras aferentes nociceptivas, con cambios microestructurales cerebrales asociados por neuroplasticidad. b) alteración del eje HHC y de todo el SE dependiente del mismo, a la cabeza los glucocorticoides, que desempeñan un papel primordial en la respuesta al estrés. c) alteración del SI, con disritmia en la secreción de citocinas pro- y antiinflamatorias, y un papel destacado de la glía, la célula local representante del SI en el cerebro y reguladora de la función neuronal asociada al dolor $\mathrm{y}, \mathrm{d}$ ) alteraciones emocionales y psicológicas, que se manifiestan como causa y efecto a la vez de las alteraciones de los tres sistemas mencionados, SN, SE y SI. Los tres juntos constituyen el supersistema de equilibrio homeostático del organismo que se encuentra alterado en el SFM sobre un terreno predispuesto genéticamente y por diversos factores de estrés, inflamatorios, infecciosos, traumáticos y psicológicos que han penetrado al supersistema por cualquiera de sus vías, especialmente en las primeras etapas de la vida, cuando el sistema se encuentra en proceso de maduración. Ellos conforman una manera de afrontamiento particular, donde destacan los rasgos de alexitimia, rabia y una personalidad relacionada con lo fóbico, frecuentemente asociados a la patología psicosomática, esto es, importantes y variadas anomalías bioquímicas y clínicas en ausencia de otra enfermedad. Podría ser que la comorbilidad psiquiátrica, ansiedad y depresión, constituyan un epifenómeno, un factor mantenedor de la enfermedad y no una causa de la misma.

Este enfoque integrador psico-neuro-inmuno-endocrinológico, no solo explica de una vez y da coherencia a tantas y tan variadas alteraciones del SFM. Además ofrece un nuevo marco de manejo y tratamiento dirigido al equilibrio de ese triángulo homeostático al que se puede acceder por diversas vías: a través de intervención psicosocial y psicoterapia de enfoque cognitivo, de biofeedback electromiográfico, de restablecimiento del ritmo circadiano, de la mejora de la calidad del sueño y otros. Con una atención muy especial a la faceta psicológica que considera el cambio de creencias, de actitud y de comportamiento como factores claves de resolución del síndrome a través de sus repercusiones neurofisiológicas, endocrinas e inmunes alteradas en esta enfermedad.

Con todo, la variable critica para el tratamiento exitoso de esta enfermedad es el cambio de creencias que tienda a la negación de lo psicológico o a la conceptualización yatrógena de que se trata de una enfermedad orgánica pura, determinada genéticamente y susceptible de ser abordada de forma simple, con tratamientos farmacológicos. Somos conscientes de que existe una paradoja difícil de salvar cognitivamente: por una parte el SFM no es una enfermedad mental ni es el producto de un conflicto psicológico, por otra es necesario un abordaje 
integral que incluya lo psicológico y que tienda a disolver las creencias relacionadas con el dolor y el sufrimiento. ¿Quién debe entonces tratar estas dolencias, los reumatólogos, los neurólogos, los psiquiatras, los inmunólogos o los psicólogos? Se trata de una paradoja que plantean los pacientes que presentan patologías que no son explicables desde el modelo orgánicopsicógeno, el modelo dual en el que siguen ancladas nuestras tradiciones médicas.

En nuestra opinión, más allá de la especialización, estos pacientes mejoran con médicos que a la vez recogen y comprenden que el SFM no es una enfermedad convencional. Ellos influyen sobre sus pacientes de manera tal que son capaces de modificar sus creencias y les llevan de la mano hacia una autocuración que desactiva las alarmas ancestrales que se pusieron en marcha precisamente por miedo al sufrimiento, por un excesivo miedo al daño. No tenemos una receta mágica que proponer, más allá de un cambio de paradigma biológico que logre superar de una vez el modelo dual. Algo que nos permitirá entender que no es necesario un conflicto psicológico para explicar determinados síntomas médicos y que al mismo tiempo logre hacer internalizar la idea de que nuestra mente es un poderosos aliado de penetración en el sistema biológico que le da soporte. Que es posible una causalidad descendente que desde la mente logre influir en el organismo entero.

\section{REFERENCIAS}

Abeles, A.M., Pillinger, M.H., Solitar, B.M., y Abeles, M. (2007). Narrative review: the pathophysiology of fibromyalgia. Annals of Internal Medicine, 146, 726-734.

Ader, R., y Cohen, N. (1975). Behaviorally conditioned immunosuppression. Psychosomatic Medicine, 3, 333340 .

Adler, G.K., y Geenen, R. (2005). Hypothalamic-pituitaryadrenal and autonomic nervous system functioning in fibromyalgia. Rheumatic Diseases Clinics of North America, 31, 187-202, xi.

Alegre de Miquel, C., y Sellas Fernández, A. (2008). Fibromyalgia: Feeling and pain. Medicina Clinica, 131, 503-504.

Alfici, S., Sigal, M., y Landau, M. (1989). Primary fibromyalgia syndrome--a variant of depressive disorder? Psychotherapy and Psychosomatics, 51, 156-161.
Arias, M. (2008). Is fibromyalgia a neurological disease? Neurología, 23, 593-601.

Barak, Y. (2006). The immune system and happiness. Autoimmunity Reviews, 58, 523-527.

Barr, A.E., Barbe, M. F., y Clark, B.D. (2004). Work-related musculoskeletal disorders of the hand and wrist: epidemiology, pathophysiology, and sensorimotor changes. The Journal of Orthopaedic and Sports Physical Therapy, 34, 610-627.

Beauchaine, T.P., Gatzke-Kopp, L., y Mead, H.K. (2007). Polyvagal Theory and developmental psychopathology: emotion dysregulation and conduct problems from preschool to adolescence. Biological Psychology, 74, 174-184.

Bengtsson, A. (2002). The muscle in fibromyalgia. Rheumatology, 41, 721-724.

Bengtsson, A., y Bengtsson, M. (1988). Regional sympathetic blockade in primary fibromyalgia. Pain, 33, 161-167.

Blalock, J.E. (2005). The immune system as the sixth sense. Journal of Internal Medicine, 257, 126-138.

Bornhauser, N.C.H. (2005). Nuevas enfermedades ¿del alma? Reflexiones psicosomáticas a propósito de algunas analogías estructurales entre síndrome de fatiga crónica, fibromialgia y sensitividad química múltiple. Revista Chilena de Neuro-Psiquiatría 43, 41-50.

Buskila, D., Press, J., y Abu-Shakra, M. (2003). Fibromyalgia in systemic lupus erythematosus: prevalence and clinical implications. Clinical Reviews in Allergy \& Immunology, 25, 25-28.

Carmona, J., Pascual, L.M., Sánchez, J.L., Maldonado, J.A., y Pereira, A. (2007). El papel de la amplificación somatosensorial en la predicción de síntomas respiratorios. Revista de Psicopatología y Psicología Clínica, 12, 15-22.

Caruso, I., Sarzi Puttini, P., Cazzola, M., y Azzolini, V. (1990). Double-blind study of 5-hydroxytryptophan versus placebo in the treatment of primary fibromyalgia syndrome. The Journal of International Medical Research, 18, 201-209.

Cloninger, C.R. (1987). A systematic method for clinical description and classification of personality variants. A proposal. Archives of General Psychiatry, 44, 573588.

Cohen, H., Buskila, D., Neumann, L., y Ebstein, R.P. (2002). Confirmation of an association between fibromyalgia and serotonin transporter promoter region (5- HTTLPR) polymorphism, and relationship to anxiety-related personality traits. Arthritis and Rheumatism, 46, 845-847.

Cohen, H., Neumann, L., Shore, M., Amir, M., Cassuto, Y., y Buskila, D. (2000). Autonomic dysfunction in patients with fibromyalgia: application of power spectral analysis of heart rate variability. Seminars in Arthritis and Rheumatism, 29, 217-227. 
Chapman, C.R., Tuckett, R.P., y Song, C.W. (2008). Pain and stress in a systems perspective: reciprocal neural, endocrine, and immune interactions. Journal of Pain, 9, 122-145.

Dadabhoy, D., Crofford, L. J., Spaeth, M., Russell, I. J., y Clauw, D. J. (2008). Biology and therapy of fibromyalgia. Evidence-based biomarkers for fibromyalgia syndrome. Arthritis Research \& Therapy, 10, 211.

Dunn, A.J. (2006). Effects of cytokines and infections on brain neurochemistry. Clinical Neuroscience Research, 6, 52-68.

Ehrlich, G.E. (2003). Pain is real; fibromyalgia isn't. The Journal of Rheumatology, 30, 1666-1667.

Emad, Y., Ragab, Y., Zeinhom, F., El-Khouly, G., AbouZeid, A., y Rasker, J.J. (2008). Hippocampus dysfunction may explain symptoms of fibromyalgia syndrome. A study with single-voxel magnetic resonance spectroscopy. The Journal of Rheumatology, 35, 13711377.

Felipe, E., y Ávila, A. (2008). Trastornos de personalidad y conducta interpersonal: Un análisis dimensional a partir del circumplex interpersonal. Revista de Psicopatología y Psicología Clínica, 13, 33-43.

Fietta, P. (2004). Fibromyalgia: state of the art. Minerva Medica, 95, 35-47, 47-52.

Furlan, R., Colombo, S., Perego, F., Atzeni, F., Diana, A., Barbic, F., et al. (2005). Abnormalities of cardiovascular neural control and reduced orthostatic tolerance in patients with primary fibromyalgia. The Journal of Rheumatology, 32, 1787-1793.

García-Bueno, B., y Leza, J.C. (2008). Inflammatory/antiinflammatory mechanisms in the brain following exposure to stress. Revista de Neurologia, 46, 675-683.

Geenen, R., Jacobs, J.W., y Bijlsma, J.W. (2002). Evaluation and management of endocrine dysfunction in fibromyalgia. Rheumatic Diseases Clinics of North America, 28, 389-404.

Germanowicz, D., Lumertz, M. S., Martínez, D., y Margarites, A.F. (2006). Sleep disordered breathing concomitant with fibromyalgia syndrome. Jornal Brasileiro de Pneumologia, 32, 333-338.

Giesecke, T., Gracely, R.H., Williams, D.A., Geisser, M.E., Petzke, F.W., y Clauw, D. J. (2005). The relationship between depression, clinical pain, and experimental pain in a chronic pain cohort. Arthritis and Rheumatism, 52, 1577-1584.

Glaser, R., y Kiecolt-Glaser, J.K. (2005). Stress-induced immune dysfunction: implications for health. Nature Reviews Immunology, 5, 243-251.

Goicoechea, A. (2008). Cerebro y dolor: esquemas en dolor neuropático. Barcelona: Ars Medica.

Graham, J. E., Christian, L. M., y Kiecolt-Glaser, J.K. (2006). Stress, age, and immune function: toward a lifespan approach. Journal of Behavioral Medicine, 29, 389-400.
Guedj, E., Cammilleri, S., Niboyet, J., Dupont, P., Vidal, E., Dropinski, J. P., et al. (2008). Clinical correlate of brain SPECT perfusion abnormalities in fibromyalgia. Journal of Nuclear Medicine, 49, 1798-1803.

Hassett, A.L., Cone, J.D., Patella, S.J., y Sigal, L.H. (2000). The role of catastrophizing in the pain and depression of women with fibromyalgia syndrome. Arthritis and Rheumatism, 43, 2493-2500.

Hazemeijer, I., y Rasker, J.J. (2003). Fibromyalgia and the therapeutic domain. A philosophical study on the origins of fibromyalgia in a specific social setting. Rheumatology, 42, 507-515.

Hudson, J. I., y Pope, H.G., Jr. (1989). Fibromyalgia and psychopathology: is fibromyalgia a form of «affective spectrum disorder»? Journal of Rheumatology Suppl, 19, 15-22.

Jacobs, J.W., Geenen, R., Evers, A.W., van Jaarsveld, C.H., Kraaimaat, F.W., y Bijlsma, J.W. (2001). Short term effects of corticosteroid pulse treatment on disease activity and the wellbeing of patients with active rheumatoid arthritis. Annals of the Rheumatic Diseases, 60 , 61-64.

Kooh, M., Martínez-Lavin, M., Meza, S., Martín-delCampo, A., Hermosillo, A.G., Pineda, C., et al. (2003). Simultaneous heart rate variability and polysomnographic analyses in fibromyalgia. Clinical and Experimental Rheumatology, 21, 529-530.

Krueger, J.M. (2008). The role of cytokines in sleep regulation. Current Pharmaceutical Design, 14, 3408-3416.

Lesch, K. P., Meyer, J., Glatz, K., Flugge, G., Hinney, A., Hebebrand, J., et al. (1997). The 5-HT transporter genelinked polymorphic region (5-HTTLPR) in evolutionary perspective: alternative biallelic variation in rhesus monkeys. Rapid communication. Journal of Neural Transmission, 104, 1259-1266.

López Ibor, J. (1966). Las neurosis como enfermedades del ánimo. Madrid: Gredos

López Ibor, J. (1972). Los equivalentes depresivos. Madrid: Paz Montalvo.

Lutz, J., Jager, L., de Quervain, D., Krauseneck, T., Padberg, F., Wichnalek, M., et al. (2008). White and gray matter abnormalities in the brain of patients with fibromyalgia: a diffusion-tensor and volumetric imaging study. Arthritis and Rheumatism, 58, 3960-3969.

Marriott, I. (2004). The role of tachykinins in central nervous system inflammatory responses. Frontiers in Bioscience, 9, 2153-2165.

Martínez-Lavin, M. (2007). Biology and therapy of fibromyalgia. Stress, the stress response system, and fibromyalgia. Arthritis Research \& Therapy, 9, 216.

Martínez-Lavin, M., Hermosillo, A.G., Rosas, M., y Soto, M.E. (1998). Circadian studies of autonomic nervous balance in patients with fibromyalgia: a heart rate variability analysis. Arthritis and Rheumatism, 41, 19661971. 
Martínez, D., y Cassol, C.M. (2008). Fibromyalgia and sleep-disordered breathing: the missing link. Arthritis Research \& Therapy, 10, 408; author reply 409.

McGowan, P.O., Sasaki, A., D’Alessio, A.C., Dymov, S., Labonte, B., Szyf, M., et al. (2009). Epigenetic regulation of the glucocorticoid receptor in human brain associates with childhood abuse. Nature Neuroscience, $12,342-348$.

Misslin, R. (2003). The defense system of fear: behavior and neurocircuitry. Clinical Neurophysiology, 33, 55-66.

Moldofsky, H. (1995). Sleep, neuroimmune and neuroendocrine functions in fibromyalgia and chronic fatigue syndrome. Advances in Neuroimmunology, 5, 39-56.

Moldofsky, H. (2008). The significance of the sleepingwaking brain for the understanding of widespread musculoskeletal pain and fatigue in fibromyalgia syndrome and allied syndromes. Joint Bone Spine, 75, 397-402.

Obal, F., Jr., y Krueger, J. M. (2004). GHRH and sleep. Sleep Medicine Reviews, 8, 367-377.

Offenbaecher, M., Bondy, B., de Jonge, S., Glatzeder, K., Kruger, M., Schoeps, P., et al. (1999). Possible association of fibromyalgia with a polymorphism in the serotonin transporter gene regulatory region. Arthritis and Rheumatism, 42, 2482-2488.

Otzenberger, H., Gronfier, C., Simon, C., Charloux, A., Ehrhart, J., Piquard, F., et al. (1998). Dynamic heart rate variability: a tool for exploring sympathovagal balance continuously during sleep in men. The American Journal of Physiology, 275, H946-950.

Peterfi, Z., Makara, G.B., Obal, F., Jr., y Krueger, J.M. (2009). The anterolateral projections of the medial basal hypothalamus affect sleep. American Journal of Physiology, 296, R1228-1238.

Porges, S.W. (1992). Vagal tone: a physiologic marker of stress vulnerability. Pediatrics, 90, 498-504.

Reiche, E.M., Morimoto, H.K., y Nunes, S.M. (2005). Stress and depression-induced immune dysfunction: implications for the development and progression of cancer. International Review of Psychiatry, 17, 515527.

Rodero, B., García-Campayo, J., Casanueva, B., y Buriel, Y. (2009). Tratamientos no farmacológicos en fibromialgia: Una revisión actual. Revista de Psicopatología y Psicología Clínica, 14, 137-151.

Rosenkranz, M. A. (2007). Substance P at the nexus of mind and body in chronic inflammation and affective disorders. Psychological Bulletin, 133, 1007-1037.

Rosenkranz, M.A., Jackson, D.C., Dalton, K.M., Dolski, I., Ryff, C.D., Singer, B.H., et al. (2003). Affective style and in vivo immune response: neurobehavioral mechanisms. Proceedings of the National Academy of Sciences of the United States of America, 100, 11148-11152.

Roy-Byrne, P., Smith, W.R., Goldberg, J., Afari, N., y
Buchwald, D. (2004). Post-traumatic stress disorder among patients with chronic pain and chronic fatigue. Psychological Medicine, 34, 363-368.

Rueda, B., y Pérez-García, A.M. (2007). Estudio de la alexitimia y de los procesos emocionales negativos en el ámbito de los factores de riesgo y la sintomatología cardiovascular. Revista de Psicopatología y Psicología Clínica, 12, 105-116.

Sayar, K., Gulec, H., Topbas, M., y Kalyoncu, A. (2004). Affective distress and fibromyalgia. Swiss Medical Weekly, 134, 248-253.

Scharf, M. B., Baumann, M., y Berkowitz, D. V. (2003). The effects of sodium oxybate on clinical symptoms and sleep patterns in patients with fibromyalgia. The Journal of Rheumatology, 30, 1070-1074.

Sharpe M.C, O'Malley P.G. (2006). Síndromes de fatiga crónica y fibromialgia. En: Levenson J. Tratado de Medicina Psicosomática (pp. 569-591). Barcelona: Ars Médica.

Sifneos, PE. (1973). The prevalence of 'alexithymic' characteristics in psychosomatic patients. Psychotherapy and Psychosomatics, 22, 255-262.

Sociedad Española de Reumatología. (2006). Reumatología Clínica, 2 (supl 1), S55-66.

Solano, C., Martínez, A., Becerril, L., Vargas, A., Figueroa, J., Navarro, C., et al. (2009). Autonomic Dysfunction in Fibromyalgia Assessed by the Composite Autonomic Symptoms Scale (COMPASS). Journal of Clinical Rheumatology 15, 172-6.

Targino, R.A., Imamura, M., Kaziyama, H.H., Souza, L.P., Hsing, W. T., Furlan, A.D., et al. (2008). A randomized controlled trial of acupuncture added to usual treatment for fibromyalgia. Journal of Rehabilitation Medicine, 40, 582-588.

Togo, F., Natelson, B. H., Adler, G. K., Ottenweller, J.E., Goldenberg, D.L., Struzik, Z.R., et al. (2009). Plasma cytokine fluctuations over time in healthy controls and patients with fibromyalgia. Experimental Biology and Medicine, 234, 232-240.

Ulas, U.H., Unlu, E., Hamamcioglu, K., Odabasi, Z., Cakci, A., y Vural, O. (2006). Dysautonomia in fibromyalgia syndrome: sympathetic skin responses and RR interval analysis. Rheumatology International, 26, 383-387.

Vgontzas, A.N., Zoumakis, M., Papanicolaou, D.A., Bixler, E. O., Prolo, P., Lin, H. M., et al. (2002). Chronic insomnia is associated with a shift of interleukin-6 and tumor necrosis factor secretion from nighttime to daytime. Metabolism: Clinical and Experimental, 51, 887-892.

Wang, H., Moser, M., Schiltenwolf, M., y Buchner, M. (2008). Circulating cytokine levels compared to pain in patients with fibromyalgia - a prospective longitudinal study over 6 months. The Journal of Rheumatology, $35,1366-1370$. 
Watkins, L.R., Hutchinson, M. R., Milligan, E.D., y Maier, S. F. (2007). «Listening» and «talking» to neurons: implications of immune activation for pain control and increasing the efficacy of opioids. Brain Research Reviews, 56, 148-169.

Watkins, L.R., y Maier, S.F. (2005). Immune regulation of central nervous system functions: from sickness responses to pathological pain. Journal of Internal Medicine, 257, 139-155.

Wieseler-Frank, J., Maier, S.F., y Watkins, L. R. (2004). Glial activation and pathological pain. Neurochemistry International, 45, 389-395.

Wieseler-Frank, J., Maier, S.F., y Watkins, L.R. (2005a). Central proinflammatory cytokines and pain enhancement. Neuro-Signals, 14, 166-174.

Wieseler-Frank, J., Maier, S.F., y Watkins, L.R. (2005b). Immune-to-brain communication dynamically modulates pain: physiological and pathological consequences. Brain, Behavior, and Immunity, 19, 104-111.
Wolfe, F., y Michaud, K. (2009). Outcome and predictor relationships in fibromyalgia and rheumatoid arthritis: evidence concerning the continuum versus discrete disorder hypothesis. The Journal of Rheumatology, 36, 831-836.

Wolfe, F., Petri, M., Alarcon, G. S., Goldman, J., Chakravarty, E.F., Katz, R.S., et al. (2009). Fibromyalgia, systemic lupus erythematosus (SLE), and evaluation of SLE activity. The Journal of Rheumatology, 36, 82-88.

Wolfe, F., Smythe, H. A., Yunus, M. B., Bennett, R. M., Bombardier, C., Goldenberg, D. L., et al. (1990). The American College of Rheumatology 1990 Criteria for the Classification of Fibromyalgia. Report of the Multicenter Criteria Committee. Arthritis and Rheumatism, 33, 160-172.

Wood, P. B., Kablinger, A. S., y Caldito, G. S. (2005). Open trial of pindolol in the treatment of fibromyalgia. The Annals of Pharmacotherapy, 39, 1812-1816. 